\title{
Direct electric stimulation to increase cerebrovascular function
}

\author{
Victor M. Pulgar ${ }^{1,2,3 *}$ \\ 'Biomedical Research and Infrastructure Center, Faculty of Natural and Physical Sciences, Winston-Salem State University, \\ Winston-Salem, NC, USA, ${ }^{2}$ Hypertension and Vascular Research Center, Wake Forest School of Medicine, Winston-Salem, \\ NC, USA, ${ }^{3}$ Department of Obstetrics and Gynecology, Wake Forest School of Medicine, Winston-Salem, NC, USA
}

Keywords: brain Stimulation, cerebral blood flow (CBF), neurovascular coupling, functional hyperemia, transcranial direct current stimulation (tDCS)

Various conditions affecting the cerebral vasculature may lead to cumulative damage and thus deterioration of brain function, in what has been called vascular cognitive impairment (Gorelick et al., 2011). Consequently, it makes sense that an increase in glucose and oxygen produced by an increase in blood flow may augment brain function. Since its rediscovery some years ago transcranial direct electric stimulation (tDCS) has attracted interest as potential therapy for patients with neurological impairments. This opinion article aims to succinctly review the mechanisms involved in neurogenic control of the cerebral blood flow (CBF) highlighting the potential of direct electrical stimulation targeting cerebral micro vessels to enhance brain function.

With the highest oxygen consumption than any other organ in the body, the brain utilizes around $20 \%$ of the total resting oxygen, making it an organ highly dependent on blood supply (Hossmann, 1994). Moreover, a direct relationship between the development of neurodegenerative diseases and impairment of CBF has been postulated (Farkas and Luiten, 2001).

The appropriate delivery of nutrients and oxygen to the brain tissue is regulated by mechanisms including cerebral autoregulation, vascular reactivity and neurovascular coupling. The autoregulatory properties of cerebral circulation make CBF independent of systemic blood pressure. Therefore, over a physiological range of pressure cerebral arteries relax when systemic pressure decreases and constrict when systemic pressure increases (Heistad and Kontos, 1983). Similarly, reactivity of the brain blood vessels to $\mathrm{pH}$ and $\mathrm{CO}_{2}$ has been suggested to link neuronal metabolic changes to cerebral blood flow.

\section{The Neurovascular Unit}

One of the unique characteristics of the brain circulation is the intimate contact between blood vessels, neurons, and glia. Thus, neurons, glia, and vascular cells are structurally and functionally related in what is called the "neurovascular unit" (Iadecola, 2004). Since brain $\mathrm{PO}_{2}$ is tightly regulated in relation to local brain activity, the neurovascular unit provides a framework for the functional interactions responsible for this concerted regulation. Thereby, functional hyperemia means that blood flow will increase in brain areas with increased activity.

From an anatomical point of view, pial arteries traveling on the surface of the brain are highly innervated with terminals coming from the peripheral nervous system (extrinsic innervation) (Hamel, 2006). These vessels are surrounded by the Virchow-Robin's space which gradually disappears as vessels enter the brain parenchyma. Cerebral arteries entering the brain parenchyma lose extrinsic innervation and come into intimate contact with neuronal and glial cells (intrinsic innervation) (Iadecola, 2004). Functions of these two vascular compartments, macro and micro vessels, involve regulation of global blood supply, as wells as control of local CBF and brain blood barrier permeability respectively. 
It is currently accepted that postsynaptic increases in $\left[\mathrm{Ca}^{++}\right]_{\mathrm{i}}$ due to activation of glutamate receptors during synaptic transmission activate the production of vasoactive mediators.

Several mediators such as neurotransmitters, adenosine, arachidonic acid metabolites, nitric oxide (NO), hydrogen and potassium, have been suggested to mediate increases in $\mathrm{CBF}$ (Iadecola, 2004).

Due to their close contact with blood vessels, astrocytes are suggested to play an important role in functional hyperemia. Astrocyte's end-feet surround brain micro capillaries, and may mediate neuron-blood vessel communication and thus neuronal activity-induced blood flow changes (Zonta et al., 2003). Vasodilatory as well as vasoconstrictor activities have been ascribed to glial cells (Metea and Newman, 2006), with an important role for glial eNOS in mediating vasodilatation (Stobart et al., 2013). New evidences also point to astrocytes as relevant components of the recently described "glymphatic pathway," an important mechanism for clearance of solutes from the brain (Iliff et al., 2012). Thus, aquaporin water channels in astrocyte's end feet would couple paravascular pathways for the vectorial convective flow of waste products from arterial toward venous routes, with solutes ultimately clearing the brain through the lymphatic system (Nedergaard, 2013).

The brain endothelium is a highly specialized tissue mediating several physiological functions, such as thrombosis, adhesion, permeability and angiogenesis (Daneman and Prat, 2015). A protective function against cerebral dysfunction has been proposed for the brain vessel's endothelium consistent with the predominant role of endothelial dysfunction in several cerebrovascular diseases. Importantly, in vivo experiments have shown that endothelial cell-derived NO mediates cortical hyperemia induced by basal forebrain electrical stimulation (Zhang et al., 1995).

Pericytes, cells located outside of the microvessels in intimate contact with endothelium and astrocyte end-feet, are more frequent on microvessels of the retina and brain and thought to regulate blood flow (Kutcher and Herman, 2009). Pericytes are considered important components of the neurovascular unit as regulators of the brain blood barrier function and also potential mediators of brain vascular dysfunction (Hamilton et al., 2010). Among the properties identified include contraction, hemostasis and angiogenesis. Given their contractile properties, pericytes may act as surrogates of smooth muscle cells in brain microvessels.

Dysfunctional interactions within the neurovascular unit have the potential to lead to brain pathophysiological alterations. Impaired endothelial cell-astrocytes or endothelial cell-pericytes signaling may cause brain blood barrier disruption (Zlokovic, 2008), whereas altered coupling between neuronal activity and vascular responses may contribute to spreading depression (Dreier, 2011).

\section{tDCS and Brain Perfusion}

Effects of electrical stimulation on the brain have been known for centuries (Priori, 2003). Work in the rat primary motor region showed that electrical stimulation may increase, decrease, or silence neuron's firing (Bindman et al., 1964; Purpura and
McMurtry, 1965). These animal studies showed that anodal stimulation caused depolarization, whereas cathodal stimulation caused hyperpolarization, thus increasing the probability for a neuron to produce an action potential. tDCS has been rediscovered as a non-invasive promising tool to modulate brain activity and as a potential treatment for psychiatric and neurological disorders (Priori, 2003; Filmer et al., 2014). An increasing number of studies have reported that tDCS modulates synaptic transmission by regulating levels of neurotransmitters such as GABA, glutamate, serotonin, and dopamine, among others (Nitsche et al., 2008).

Reports showing that stimulation of cerebellar neurons increased diameter of both adjacent arterioles and the upstream vessels, provided a demonstration of the propagation of vascular responses induced by increased neural activity (Iadecola et al., 1997). Importantly, cerebellar stimulation at the fastigial nucleus, reduced ischemia induced by medial cerebral artery occlusion in rats through NO-mediated hemodynamic mechanisms (Zhang and Iadecola, 1993).

tDCS in humans is performed by applying direct current over the scalp using electrodes and its effects depend on the size, polarity and position of the electrodes, current intensity, duration of stimulation, and tissue properties (DaSilva et al., 2011). Given the intimate relationship between neuronal activity and CBF, it is expected that tDCS will increase brain perfusion, as shown in animal (Han et al., 2014) and human (Zheng et al., 2011) studies. The opinion presented in this article is that in addition to the changes in neuronal-derived metabolites, evidences showing responses to electrical stimulation in non-neuronal cells suggest that tDCS acting on these cells has also the potential to modulate brain perfusion (Figure 1). Thus, understanding the vascular effects of tDCS may improve the treatment of diseases associated with vascular dysfunction.

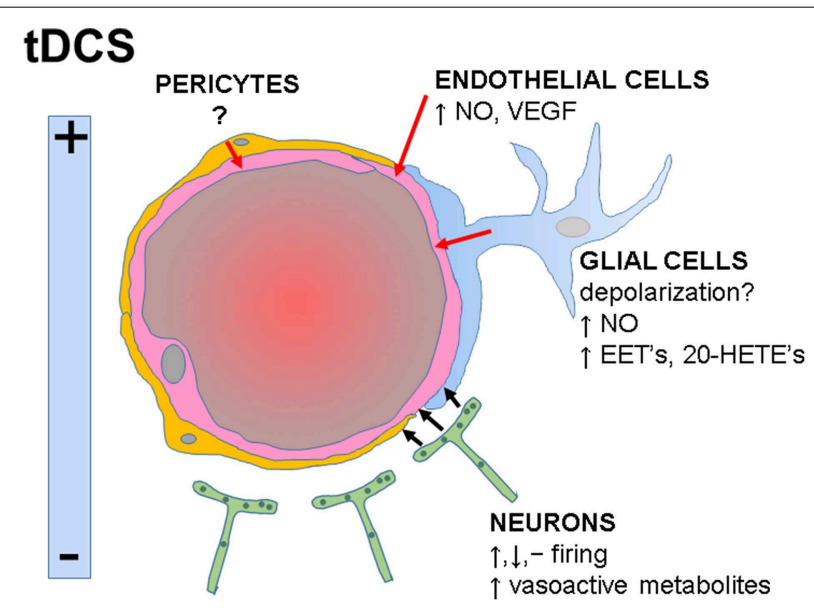

FIGURE 1 | Functional interactions within the neurovascular unit and potential influences of tDCS on specific cell types. tDCS-induced electrical fields may increase, decrease or have no effects on neuronal firing rates. Neuronal-derived metabolites directly or indirectly activate endothelial cells (black arrows), inducing vasodilatation. Potential direct effects of tDCS on glial, endothelial cells, and pericytes are indicated (red arrows). NO, nitric oxide; VEGF, vascular endothelial growth factor; EET's, epoxyeicosatrienoic acids; 20-HETE's, 20-hydroxyeicosatetraeonic acids. 
Direct effects of electric stimulation on neurons in vitro include alignment of neurites perpendicular to the electric field, increased growth and migration (Pan and Borgens, 2012). In mouse coronal slices, a role for electrical stimulation-induced synaptic plasticity was demonstrated, an effect that may underlie implications of tDCS on motor learning (Fritsch et al., 2010). Results obtained in rat brain slices suggested that electrical stimulation modulates long term potentiation in a polarity-specific manner supporting a regulatory role of tDCS on synaptic plasticity (Ranieri et al., 2012).

Described effects of electrical stimulation on astrocytes in vitro include changes in metabolism depending on field polarization and applied voltage (Huang et al., 1997), as well as migration and perpendicular alignment (Pelletier et al., 2014). A theoretical analysis concluded that $\mathrm{tDCS}$ has the potential to directly stimulate glial cells since the tDCS-induced changes in membrane potential are similar to the changes induced in astrocytes during neuronal activation (Ruohonen and Karhu, 2012).

The effects of electric stimulation on endothelial cells in vitro include the alignment perpendicular to the direction of the electrical field, migration, and elongation (Zhao et al., 2012). These effects are associated with increases in VEGF production, suggesting that electrical stimulation may modulate angiogenesis (Zhao et al., 2012). Conversely, the data from brain slices, including effects on synaptic plasticity (Fritsch et al., 2010) are obtained in the absence of circulation, which may indicate that there is no endothelial contribution to the neuronal effects of electric stimulation. However, endothelial cells in culture exposed to a low physiological electrical field $(3.3 \mathrm{mV} / \mathrm{mm})$ showed increased NO production (Trivedi et al., 2013), suggesting a direct route by which electric stimulation may increase brain perfusion. Modeling of the electric properties of the brain suggests that the electric field generated during tDCS in humans is around $1 \mathrm{mV} / \mathrm{mm}$ (Neuling et al., 2012) indicating that endothelial cell-dependent responses may be triggered during tDCS.

The proposed role of pericytes in the neurovascular unit suggests that pericytes may transduce signals from neurons to endothelial cells (Hall et al., 2014). Thus, during neuronal activation glutamate release produces prostaglandin $E_{2}$ which in turn will induce capillaries vasodilatation by activating $\mathrm{K}^{+}$currents in pericytes. These are excitable cells and a direct effect of tDCS on pericyte's membrane potential may hyperpolarize it and induce vasodilatory signals. Whether pericyte-mediated responses to $\mathrm{tDCS}$ are playing a role in the tDCS effects remains to be elucidated.

Although general agreement has been observed between animal and human studies (Bennabi et al., 2014), it is necessary to note that stimulating parameters used in animal in vivo and in in vitro protocols are higher than those used in humans where a maximum current density of $\sim 0.28 \mathrm{~A} / \mathrm{m}^{2}$ is used (Im et al., 2012). In contrast a maximum safe stimulation in rats was reported at 142.9 A/m² (Liebetanz et al., 2009).

\section{tDCS and Augmentation of Brain Function}

In humans, evidences indicate the potential of tDCS to increase cognitive, motor and memory function. For example, tDCS may enhance gesture comprehension by improving gesture and language integration (Cohen-Maximov et al., 2014), a result especially relevant in cases of autism where patients have difficulties processing symbolic gestures (Baron-Cohen, 1988). Anodal tDCS administered repeatedly facilitates language (Meinzer et al., 2014) and motor skill learning (Zimerman et al., 2013). tDCS has also been shown to produce long-lasting effects on number processing (Cohen Kadosh et al., 2010) and there is increasing interest in the applicability of tDCS for memory enhancement (Bennabi et al., 2014).

However, not all tDCS studies have observed positive effects. Thus, the rate of motor sequence learning is increased by anodal tDCS and decreased by cathodal stimulation, whereas tDCS applied prior to the motor task slowed learning (Stagg et al., 2011). Also cerebellar tDCS has been shown to impair practice-dependent improvement in a working memory task (Ferrucci et al., 2008), whereas tDCS applied to pre-frontal cortex disrupts sensory-motor training (Filmer et al., 2013). Clearly, the research describing the efficacy of tDCS for motor and cognitive improvement is still inconclusive.

Brain blood flow responses to specific tasks may involve cortical and subcortical structures, as seen in the attention-derived effects on flow in both the visual cortex and the lateral geniculate nucleus (O'Connor et al., 2002). Thus, it is relevant to point out that tDCS may also modulate blood flow in subcortical structures (Lang et al., 2005; Nonnekes et al., 2014) demonstrating broader effects of $\mathrm{tDCS}$ on $\mathrm{CBF}$.

Recent technical developments applying tDCS simultaneously with electroencephalography and brain blood flow measurements (Dutta et al., 2015) will help to integrate tDCS with modulation of vascular function and ultimately changes in human behavior.

\section{Perspectives and Future Directions}

In order to expand direct therapeutic applicability, tDCS needs to overcome the challenges related to inter- and intra-subject variability and parameters of stimulation impacting neuroplasticity (i.e., short $v s$. long term stimulation), among others. Simultaneous determination of vascular signals and cognitive performance during tDCS will help to integrate electrical stimulation with vascular functioning and changes in behavior. The relationships between neuronal and vascular effects are complex and it is proven difficult to differentiate between the effects of electrical stimulation on those two tissues, it is however conceivable that in addition to the vascular effects of neuronal-derived metabolites, direct effects of tDCS on nonneuronal cells especially glial and endothelial cells modulate brain perfusion. Thus, a deeper understanding of the effects tDCS have on non-neuronal members of the neurovascular unit is essential.

\section{Acknowledgments}

The author thanks Anne Jeffers for language revision of the article. 


\section{References}

Baron-Cohen, S. (1988). Social and pragmatic deficits in autism: cognitive or affective? J. Autism Dev. Disord. 18, 379-402. doi: 10.1007/BF02212194

Bennabi, D., Pedron, S., Haffen, E., Monnin, J., Peterschmitt, Y., and Van Waes, V. (2014). Transcranial direct current stimulation for memory enhancement: from clinical research to animal models. Front. Syst. Neurosci. 8:159. doi: 10.3389/fnsys.2014.00159

Bindman, L. J., Lippold, O. C. J., and Redfearn, J. W. T. (1964). The action of brief polarizing currents on the cerebral cortex of the rat (1) during current flow and (2) in the production of long-lasting after-effects. J. Physiol. 172, 369-382. doi: 10.1113/jphysiol.1964.sp007425

Cohen Kadosh, R., Soskic, S., Iuculano, T., Kanai, R., and Walsh, V. (2010). Modulating neuronal activity produces specific and long-lasting changes in numerical competence. Curr. Biol. 20, 2016-2020. doi: 10.1016/j.cub.2010.10.007

Cohen-Maximov, T., Avirame, K., Flõel, A., and Lavidor, M. (2014). Modulation of gestural-verbal semantic integration by tDCS. Brain Stimul. doi: 10.1016/j.brs.2014.12.001. [Epub ahead of print]

Daneman, R., and Prat, A. (2015). The Blood-Brain Barrier. Cold Spring Harb. Perspect. Biol. 7:a020412. doi: 10.1101/cshperspect.a020412

DaSilva, A. F., Volz, M. S., Bikson, M., and Fregni, F. (2011). Electrode positioning and montage in transcranial direct current stimulation. J. Vis. Exp. 51:e2744. doi: $10.3791 / 2744$

Dreier, J. P. (2011). The role of spreading depression, spreading depolarization and spreading ischemia in neurological disease. Nat. Med. 17, 439-447. doi: $10.1038 / \mathrm{nm} .2333$

Dutta, A., Jacob, A., Chowdhury, S., Das, A., and Nitsche, M. (2015). EEGNIRS based assessment of neurovascular coupling during anodal transcranial direct current stimulation - a stroke case series. J. Med. Syst. 39, 1-9. doi: 10.1007/s10916-015-0205-7

Farkas, E., and Luiten, P. G. M. (2001). Cerebral microvascular pathology in aging and Alzheimer's disease. Prog. Neurobiol. 64, 575-611. doi: 10.1016/S03010082(00)00068-X

Ferrucci, R., Marceglia, S., Vergari, M., Cogiamanian, F., Mrakic-Sposta, S., Mameli, F., et al. (2008). Cerebellar transcranial direct current stimulation impairs the practice-dependent proficiency increase in working memory. J. Cogn. Neurosci. 20, 1687-1697. doi: 10.1162/jocn.2008.20112

Filmer, H. L., Dux, P. E., and Mattingley, J. B. (2014). Applications of transcranial direct current stimulation for understanding brain function. Trends Neurosci. 37, 742-753. doi: 10.1016/j.tins.2014.08.003

Filmer, H. L., Mattingley, J. B., Marois, R., and Dux, P. E. (2013). Disrupting prefrontal cortex prevents performance gains from sensory-motor training. J. Neurosci. 33, 18654-18660. doi: 10.1523/JNEUROSCI.2019-13.2013

Fritsch, B., Reis, J., Martinowich, K., Schambra, H. M., Ji, Y., Cohen, L. G., et al. (2010). Direct current stimulation promotes BDNF-dependent synaptic plasticity: potential implications for motor learning. Neuron 66, 198-204. doi: 10.1016/j.neuron.2010.03.035

Gorelick, P. B., Scuteri, A., Black, S. E., DeCarli, C., Greenberg, S. M., Iadecola, C., et al. (2011). Vascular contributions to cognitive impairment and dementia: a statement for healthcare professionals from the American Heart Association/American Stroke Association. Stroke 42, 2672-2713. doi: 10.1161/STR.0b013e3182299496

Hall, C. N., Reynell, C., Gesslein, B., Hamilton, N. B., Mishra, A., Sutherland, B. A., et al. (2014). Capillary pericytes regulate cerebral blood flow in health and disease. Nature 508, 55-60. doi: 10.1038/nature 13165

Hamel, E. (2006). Perivascular nerves and the regulation of cerebrovascular tone. J. Appl. Physiol. 100, 1059-1064. doi: 10.1152/japplphysiol.00954.2005

Hamilton, N. B., Attwell, D., and Hall, C. N. (2010). Pericyte-mediated regulation of capillary diameter: a component of neurovascular coupling in health and disease. Front. Neuroenergetics 2:5. doi: 10.3389/fnene.2010.00005

Han, C. H., Song, H., Kang, Y. G., Kim, B. M., and Im, C. H. (2014). Hemodynamic responses in rat brain during transcranial direct current stimulation: a functional near-infrared spectroscopy study. Biomed. Opt. Express 13, 1812-1821. doi: 10.1364/BOE.5.001812

Heistad, D., and Kontos, H. (1983). Handbook of Physiology: The Cardiovascular System III. Bethesda, MD: American Physiological Society.

Hossmann, K. A. (1994). Viability thresholds and the penumbra of focal ischemia. Ann. Neurol. 36, 557-565. doi: 10.1002/ana.410360404
Huang, R., Peng, L., and Hertz, L. (1997). Effects of a low-voltage static electric field on energy metabolism in astrocytes. Bioelectromagnetics 18, 77-80. doi: 10.1002/(SICI)1521-186X(1997)18:1<77::AID-BEM11>3.0.CO;2-N

Iadecola, C. (2004). Neurovascular regulation in the normal brain and in Alzheimer's disease. Nat. Rev. Neurosci. 5, 347-360. doi: 10.1038/nrn1387

Iadecola, C., Yang, G., Ebner, T., and Chen, G. (1997). Local and propagated vascular responses evoked by focal synaptic activity in cerebellar cortex. Neurophysiol 78, 651-659.

Iliff, J. J., Wang, M., Liao, Y., Plogg, B. A., Peng, W., Gundersen, G. A., et al. (2012). A paravascular pathway facilitates CSF flow through the brain parenchyma and the clearance of interstitial solutes, including amyloid $\beta$. Sci. Transl. Med. 4:147ra111. doi: 10.1126/scitranslmed.3003748

Im, C. H., Park, J. H., Shim, M., Chang, W. H., and Kim, Y. H. (2012). Evaluation of local electric fields generated by transcranial direct current stimulation with an extracephalic reference electrode based on realistic 3D body modeling. Phys. Med. Biol. 57:2137. doi: 10.1088/0031-9155/57/8/2137

Kutcher, M. E., and Herman, I. M. (2009). The pericyte: cellular regulator of microvascular blood flow. Microvasc. Res. 77, 235-246. doi: 10.1016/j.mvr.2009.01.007

Lang, N., Siebner, H. R., Ward, N. S., Lee, L., Nitsche, M. A., Paulus, W., et al. (2005). How does transcranial DC stimulation of the primary motor cortex alter regional neuronal activity in the human brain? Eur. J. Neurosci. 22, 495-504. doi: 10.1111/j.1460-9568.2005.04233.x

Liebetanz, D., Koch, R., Mayenfels, S., König, F., Paulus, W., and Nitsche, M. A. (2009). Safety limits of cathodal transcranial direct current stimulation in rats. Clin. Neurophysiol. 120, 1161-1167. doi: 10.1016/j.clinph.2009.01.022

Meinzer, M., Jähnigen, S., Copland, D. A., Darkow, R., Grittner, U., Avirame, K., et al. (2014). Transcranial direct current stimulation over multiple days improves learning and maintenance of a novel vocabulary. Cortex 50, 137-147. doi: 10.1016/j.cortex.2013.07.013

Metea, M. R., and Newman, E. A. (2006). Glial cells dilate and constrict blood vessels: a mechanism of neurovascular coupling. J. Neurosci. 26, 2862-2870. doi: 10.1523/JNEUROSCI.4048-05.2006

Nedergaard, M. (2013). Garbage truck of the brain. Science 340, 1529-1530. doi: $10.1126 /$ science. 1240514

Neuling, T., Wagner, S., Wolters, C. H., Zaehle, T., and Herrmann, C. S. (2012) Finite element model predicts current density distribution for clinical applications of tDCS and tACS. Front. Psychiatry 3:83. doi: 10.3389/fpsyt.2012. 00083

Nitsche, M. A., Cohen, L. G., Wassermann, E. M., Priori, A., Lang, N., Antal, A., et al. (2008). Transcranial direct current stimulation: State of the art 2008. Brain Stimul. 1, 206-223. doi: 10.1016/j.brs.2008.06.004

Nonnekes, J., Arrogi, A., Munneke, M. A. M., van Asseldonk, E. H. F., Oude Nijhuis, L. B., Geurts, A. C., et al. (2014). Subcortical structures in humans can be facilitated by transcranial direct current stimulation. PLoS ONE 9:e107731. doi: 10.1371/journal.pone.0107731

O'Connor, D. H., Fukui, M. M., Pinsk, M. A., and Kastner, S. (2002). Attention modulates responses in the human lateral geniculate nucleus. Nat. Neurosci. 5, 1203-1209. doi: 10.1038/nn957

Pan, L., and Borgens, R. B. (2012). Strict perpendicular orientation of neural crestderived neurons in vitro is dependent on an extracellular gradient of voltage. J. Neurosci. Res. 90, 1335-1346. doi: 10.1002/jnr.22809

Pelletier, S. J., Lagacé, M., St-Amour, I., Arsenault, D., Cisbani, G., Chabrat, A., et al. (2014). The morphological and molecular changes of brain cells exposed to direct current electric field stimulation. Int. J. Neuropsychopharmacol. 18. doi: 10.1093/ijnp/pyu090

Priori, A. (2003). Brain polarization in humans: a reappraisal of an old tool for prolonged non-invasive modulation of brain excitability. Clin. Neurophysiol. 114, 589-595. doi: 10.1016/S1388-2457(02)00437-6

Purpura, D. P., and McMurtry, J. G. (1965). Intracellular activities and evoked potential changes during polarization of motor cortex. J. Neurophysiol. 28, $166-185$.

Ranieri, F., Podda, M. V., Riccardi, E., Frisullo, G., Dileone, M., Profice, P., et al. (2012). Modulation of LTP at rat hippocampal CA3-CA1 synapses by direct current stimulation. J. Neurophysiol. 107, 1868-1880. doi: 10.1152/jn.00319.2011

Ruohonen, J., and Karhu, J. (2012). tDCS possibly stimulates glial cells. Clin. Neurophysiol. 123, 2006-2009. doi: 10.1016/j.clinph.2012.02.082 
Stagg, C. J., Jayaram, G., Pastor, D., Kincses, Z. T., Matthews, P. M., and JohansenBerg, H. (2011). Polarity and timing-dependent effects of transcranial direct current stimulation in explicit motor learning. Neuropsychologia 49, 800-804. doi: 10.1016/j.neuropsychologia.2011.02.009

Stobart, J. L. L., Lu, L., Anderson, H. D. I., Mori, H., and Anderson, C. M. (2013). Astrocyte-induced cortical vasodilation is mediated by D-serine and endothelial nitric oxide synthase. Proc. Natl. Acad. Sci. U.S.A. 110, 3149-3154. doi: 10.1073/pnas. 1215929110

Trivedi, D. P., Hallock, K. J., and Bergethon, P. R. (2013). Electric fields caused by blood flow modulate vascular endothelial electrophysiology and nitric oxide production. Bioelectromagnetics 34, 22-30. doi: 10.1002/bem. 21741

Zhang, F., and Iadecola, C. (1993). Fastigial stimulation increases ischemic blood flow and reduces brain damage after focal ischemia. J. Cereb. Blood Flow Metab. 13, 1013-1019. doi: $10.1038 /$ jcbfm.1993.127

Zhang, F., Xu, S., and Iadecola, C. (1995). Role of nitric oxide and acetylcholine in neocortical hyperemia elicited by basal forebrain stimulation: evidence for an involvement of endothelial nitric oxide. Neuroscience 69, 1195-1204. doi: 10.1016/0306-4522(95)00302-Y

Zhao, Z., Qin, L., Reid, B., Pu, J., Hara, T., and Zhao, M. (2012). Directing migration of endothelial progenitor cells with applied DC electric fields. Stem Cell Res. 8, 38-48. doi: 10.1016/j.scr.2011.08.001
Zheng, X., Alsop, D. C., and Schlaug, G. (2011). Effects of transcranial direct current stimulation (tDCS) on human regional cerebral blood flow. Neuroimage 58, 26-33. doi: 10.1016/j.neuroimage.2011.06.018

Zimerman, M., Nitsch, M., Giraux, P., Gerloff, C., Cohen, L. G., and Hummel, F. C. (2013). Neuroenhancement of the aging brain: restoring skill acquisition in old subjects. Ann. Neurol. 73, 10-15. doi: 10.1002/ana.23761

Zlokovic, B. V. (2008). The blood-brain barrier in health and chronic neurodegenerative disorders. Neuron 57, 178-201. doi: 10.1016/j.neuron.2008.01.003

Zonta, M., Angulo, M. C., Gobbo, S., Rosengarten, B., Hossmann, K. A., and Pozzan, T., et al. (2003). Neuron-to-astrocyte signaling is central to the dynamic control of brain microcirculation. Nat. Neurosci. 6, 43-50. doi: 10.1038/nn980

Conflict of Interest Statement: The authors declare that the research was conducted in the absence of any commercial or financial relationships that could be construed as a potential conflict of interest.

Copyright $\odot 2015$ Pulgar. This is an open-access article distributed under the terms of the Creative Commons Attribution License (CC BY). The use, distribution or reproduction in other forums is permitted, provided the original author(s) or licensor are credited and that the original publication in this journal is cited, in accordance with accepted academic practice. No use, distribution or reproduction is permitted which does not comply with these terms. 